\title{
The Measurement of Auditory Abilities of Blind, Partially Sighted, and Sighted Children
}

\author{
Lazar Stankov and Georgina Spilsbury \\ University of Sydney
}

A battery of 26 auditory tests was given to groups of 30 blind, partially sighted, and sighted children. Primary factors defined by the tests corresponded closely to those previously found with a similar battery (Stankov \& Horn, in press). Overall, the blind and sighted were equal on most of the abilities measured by the tests; however, differences could be observed if particular primaries were considered. Blind children performed better on tests measuring tonal memory but worse on tests of masking and rhythm. The partially sighted group demonstrated poorer performance than the other two groups; this was attributed to possible cognitive and/or personality problems in addition to those associated with reduced vision.

Auditory abilities have been studied since the beginnings of experimental psychology, but two papers by Karlin $(1941,1942)$ can be viewed as landmarks for the approach to be adopted here. In these papers Karlin used the Thurstonian method of factor analysis in order to analyze correlations among a large number of auditory tests: He was interested in discovering primary auditory mental abilities. His tests can be roughly divided into two groups. The first group he selected from a field already more than 20 years old-the field of "musical abilities." Seashore pioneered the research in this area; and Wing, Drake, Bentley, and many others followed his

APPLIED PSYCHOLOGICAL MEASUREMENT Vol. 2, No. 4 Fall 1978 pp. 491-503

(c) Copyright 1978 West Publishing Co. lead (see Shuter, 1968). Karlin also employed several tests which subsequently became a part of "speech perception" studies (Harris, 1964; Solomon, 1959).

It would appear from the foregoing that auditory tests need to be a part of a broader field of research on human abilities than can generally be found. Careful reading of source papers on various theories about the structure of abilities shows that they all have a place reserved for auditory abilities; but only one of them, the theory of fluid (Gf) and crystallized (Gc) intelligence (Horn, 1974), incorporated them directly.

The way in which auditory abilities found their place within the Gf/Gc theory illustrates the use of a factor analytic theory in a predictive sense. Horn (1968) claimed that consistent emergence of a General Visualization (Gv) factor in addition to Gf and Gc implies that should one use another (nonvisual) modality for inputting information, a broad perceptual factor reflecting that sensory input would emerge. Stankov (1971) attempted to test the above contention within the auditory domain. In that study he assembled a battery of tests from the areas of musical abilities and speech perception, as well as from visual $\mathrm{Gf}, \mathrm{Gc}$, and $\mathrm{Gv}$. In addition, he utilized auditory stimuli in order to construct new tests involving processes typical of $\mathrm{Gf}, \mathrm{Gc}$, and Gv. Altogether, 72 tests were given to a group of 241 adults. At the second-order analysis, he 
found $\mathrm{Gf}$ and $\mathrm{Gc}$, as expected, and a third factor representing a General Auditory function $(\mathrm{Ga})$.

The inclusion of auditory tests within the batteries of cognitive abilities has both theoretical and practical implications. On the theoretical side, it appears that at least four new primaries should be added to the list of cognitive abilities (Stankov \& Horn, in press). One of those, Temporal Tracking $(\mathrm{Tc})$, represents a new Gf primary reflecting the ability to manipulate and detect changes among sequentially presented stimuli. Of the other three factors, Discrimination Among Sound Patterns (DASP) loads on Gf, Gc, and $\mathrm{Ga}$; and Speech Perception Under Distriction/Distortion (SPUD) and Maintaining and Judging Rhythm (MaJR) are clear Ga primaries.

On the practical side, the assessment of Gf and $\mathrm{Gc}$ with auditory tests opens the possibility for testing intellectual abilities of visually deprived groups. The aims of the present paper are to look more closely at that possibility and to verify earlier findings. First, however, some background of what is presently known about auditory capabilities of the blind will be useful.

\section{Auditory Abilities of the Blind}

The popular "compensation hypothesis," which states that the other senses of the blind become "quicker," is not endorsed by those who have investigated the issue (Hayes, 1941). More accurately, the blind probably utilize their intact sensory channels to a fuller capacity (Rice, 1970). The auditory channel has been the most popular channel studied, and it has been found that there is no significant difference between blind and sighted on a number of variables. Following are some of the variables on which differences were found between blind and sighted subjects. The blind were found to excel on auditory localization (Rice, 1970); Wing's tests of Chord Analysis, Pitch Change, and Tonal Memory (Pitman, 1965); Drake's test of Memory for Melody (Drake, 1954); and Seashore's test of Loudness Discrimination. However, they showed inferior performance on Seashore's tests of Pitch
Discrimination, Tonal Memory, and Rhythm (Juurmaa, 1967). It is worth noting that most variables on which differences occurred belong to the General Auditory function $(\mathrm{Ga})$.

The foregoing account of differences implies a correlation between the degree of vision and these variables. It must be mentioned, however, that in most of the studies quoted, degree of vision was not properly controlled. Typically, no distinction was made between totally blind and those with light perception. The other point which should be made clear is that differences between means do not tell anything about differences between covariance matrices or about factor structure. Only one study (Juurmaa, 1967) looked at that particular question and concluded that "blindness is not in itself a factor hindering differentiation of the ability structure."

Numerous studies with the blind were devoted to attempts to devise adequate tests for measuring their intellective abilities. Most efforts were directed at developing nonverbal tests, mainly by constructing tactual analogues of visual tests such as Progressive Matrices (see Morse, 1971, for a review). The problem with this approach is that, to a large extent, tactual (haptic) tests usually involve undesirable kinaesthetic and tactual components. Auditory tests which are capable of measuring Gf adequately should represent a considerable improvement over the present situation.

\section{The Aim of the Present Study}

The previous sections of this paper have focused on two related topics. Firstly, it was emphasized that the findings of Stankov and Horn (in press) should be replicated. Secondly, differences would be expected between blind, partially sighted, and sighted groups. Although the nature of these differences for each variable can be specified, they will not be itemized here. It should be emphasized, however, that these differences must be differences in a multivariate sense, because it was implied above that all 
variables are intercorrelated. The main concern here is to compare covariance matrices and the mean vectors, the null-hypothesis being that these are equal to each other. Mutivariate analysis of variance (MANOVA) is an appropriate technique for this purpose.

\section{Method}

\section{Tests}

With the exception of Test 3 (Dichotic Listening), the tests were chosen from among those used in the Stankov and Horn (in press) study. Because the tests were to be administered to blind subjects who would type their answers in braille, all of the tests except Number and Letter Span, Tonal and Letter Reordering, Rapid Spelling, and Cloze were made into multiple-choice format. For the latter two tests the blind persons were permitted to use standard braille contractions in punching the words. Following is a description of the tests which were used:

1-2. Memory Span. Tests from French, Ekstrom, and Price (1963).

3. Dichotic Listening (18 items). Six digits were given in pairs, three to the left ear and three to the right ear; the subjects wrote down the digits in order, giving left ear first, then right ear.

4-5. Tonal and Letter Reordering (21 items). Numbers were assigned to the three notes (letters), and the testees wrote down the order in which they occurred in the second presentation. Response: 312,231 , and so forth.

6. Tonal Figures (21 items). The testees chose the set of four notes which contained the same four notes as in the first set, but played in the opposite order, selecting from four alternatives.

7-8. Tonal and Chord Series (21 items). Examinees determined which of the three notes (chords) continued the sequence established in the first four.
9. Vocabulary (24 items). Subjects chose the synonym of a spoken word from among five alternatives, with words ranging from easy to difficult.

10. Cloze (21 sentences). Two words from an eight-word sentence were deleted; subjects had to write down words which would make the sentence grammatically acceptable.

11. Rapid Spelling (21 items). Words were spelled rapidly as subjects wrote them down. Blind subjects were allowed to use contractions.

12. Chord Analysis (15 items). Subjects determined how many notes were played in a chord (Wing, 1966).

13. Pitch Change (21 items). Two chords were played, one after the other; in the second playing, one note might have differed. Subjects stated whether the chords were the same or, if changed, whether a note moved up or down (Wing, 1966).

14. Tonal Memory (21 items). Pairs of tunes 3 to 10 notes long were presented one after the other. Subjects determined which note had changed in the second playing (Wing, 1966).

15. Chord Decomposition (21 items). A chord followed by three sets of three notes was presented. Subjects determined which set contained the same notes as those of the chord.

16. Tonal Memory (21 items). Pairs of tonal sequences were presented. Each sequence consisted of 3,4 , or 5 tones. Subjects determined which note differed in the second playing (Seashore, Lewis, \& Saetveit, 1960).

17. Musical Memory (19 items-four possible answers). Subjects were to match subsequent melodies with the first melody and to indicate if they were the same, in a different key, or if a note or time were different (Drake, 1954). 
18. Chord Parts Decomposition (21 items). A chord composed of three notes was presented; then three alternatives were given. Each alternative was a two-note interval. Subjects were to indicate which one of the alternatives contained two notes from the first chord.

19. Sound Blending (21 items). A word was sounded part by part. Subjects were to choose the word from among four phonetically balanced words.

20-21. Expanded and Compressed Speech (21 items). A word was played at slower (faster) speed. Subjects were to identify the word from among four choices.

22-23. Noise and Talk Masking (35 items). Subjects were to pick out a single word from continuous background talking (or noise) and then choose one of four alternatives as being the same as the masked word.

24. Rhythm (21 items). Subjects determined whether two rhythmic patterns were the same or different (Seashore et al., 1960).

25. Tempo "A" (10 items). Subjects were to continue to count a beat established by a metronome during silence until told to stop. Score: number of beats different from norms, with a high score indicating poor performance (Drake, 1954).

26. Tempo "B." Same test as 25 except that an interfering beat was introduced in place of the silent interval (Drake, 1954).

\section{Subjects}

The subjects were children aged between 10 and 15 years who had no known hearing impairment. The blind children consisted of virtually all blind children of that age attending special schools for the blind in the Sydney metropolitan area. Fourteen of the children were congenitally blind; two had become blind at three and five years of age, respectively; and the rest had residual vision, ranging from light to form-and-motion perception. Children were classified as blind if they used braille in their school work. (Some of these children may have had usable sight, but it is extremely difficult to tell how much use a blind person makes of his residual vision.) In all cases the braille-using group had been rated a maximum of $6 / 60$ in the better eye.

The partially sighted children were 19 pupils from the partially seeing unit of a high school and 11 boys attending a school for blind children. The vision of this group ranged from $60 / 60$ to $6 / 60$, and all could read large print. The sighted sample was drawn from the two high schools located in the same general area as the school for blind. Where possible, previous IQ scores were obtained (the verbal IQ of the blind group is either from WISC, Otis CD, or Williams Intelligence Scale). It should be noted, however, that the IQ mean for the partially sighted group (around 95) was lower by 10 IQ points than that for the other two groups. The final sample consisted of 30 children from each group.

\section{Testing Procedure}

The tests were recorded and played on a fourtrack tape recorder. Each child wore a set of stereo earphones through which the items were administered. The battery took between four and six hours to administer, depending on how quickly the group grasped the instructions. The tests were given to groups ranging between 7 and 12 children and in sessions of between one and two hours duration, depending on the schools' routines. Blind children used braille typewriters to record their answers, punching both the item number and their answer choice. The other two groups were provided with sheets containing item numbers and a blank space in which they had to write the selected alternative.

Another important feature of the present test battery should be mentioned here. For most tests, a third of the items were given to the left ear, a third to the right ear, and the rest to both ears together. These conditions of presentation 
obviously allowed for a separate study of ear differences. This will not be covered in the present paper, however, since the analyses have not yet been completed. For this study, the total scores (summed over all conditions employed) were used on the assumption that they were more reliable and that they would provide the best approximation to the free-room situation in which most auditory tests are likely to be used (e.g., Stankov, 1971).

\section{Statistical Analysis}

For the purpose of replicating previous findings (Stankov, 1971; Stankov \& Horn, in press), a method of factor analysis called Little Jiffy, Mark IV was used (see Kaiser \& Rice, 1974). This method is based on image analysis and employs orthoblique rotations. Detailed description of the program is provided in the reference quoted above, but in order to make subsequent discussions clear, two points must be emphasized. First, this program's criteria for salience of factor loadings differ from those typically used in factor analysis. Salient loadings are not those which exceed in absolute value some arbitrary cut-off point-they are related to the anti-image variances. Because of this feature, it is possible that some loadings which have relatively high values would be considered nonsalient. In order to avoid confusion and clutter, a so-called conventionally scaled factor-pattern matrix is presented in Table 2 with only salient loadings included. This principle was not followed with the second-order factor of Table 3.

Second, the procedure for calculating factor scores is also different from that typically employed in factor analysis. An interesting feature of this procedure is that factor score intercorrelations are exactly the same as factor intercorrelations. Factor scores are transformed to yield a mean of 500 and a standard deviation of 100 . For the purpose of testing the differences between the groups, the MANOVA program of Cooley and Lohnes (1971) was employed.

\section{Results}

\section{The Problem of Replication- Factor Analysis of Auditory Tests}

First order analysis. The present study is one of a series designed to explore the auditory domain. The studies of Stankov (1971, 1978), Horn (1974), and Stankov and Horn (in press) employed basically the same tests. Factor labels used throughout this paper follow those of Stankov and Horn (in press). In Stankov (1978) and Horn (1974) sub-samples of variables from the other two studies above were used; the labels for the factors were narrow in order to reflect closely the processes implied by the variables chosen. Table 1 presents factor labels employed in different studies.

The result of factor analysis of 26 tests (based on 90 subjects) is presented in Table 2. Not every factor will be elaborated upon here, since their nature can easily be grasped by considering the tests which define them. As in the Stankov (1971) and Stankov and Horn (in press) studies, this experiment yielded seven first-order factors. Of these factors, Temporal Tracking (Tc, Factor II), Speech Perception Under Distortion/Distraction (SPUD, Factor V), and Maintaining and Judging Rhythm (MaJR, Factor VI) have been replicated. Two other factors have been replicated, but in a somewhat different sense. These are Auditory Immediate Memory (Msa, Factor I) and Discrimination Among Sound Patterns (DASP, Factor IV).

The original Msa of Stankov and Horn (in press) was broader than Factor $I$ in the present study, since the former factor included such tests as Tonal Figures. Factor I also differs from the original in that it involves a verbal component (Tests 5, 10, and 23). Similarly, DASP seemed to comprise two types of variables. The present result identifies one of these groups (which had higher loadings than the other group) as tonal memory. It is rather similar to Horn's (1974) Nonsymbolic Recognition Memory. 


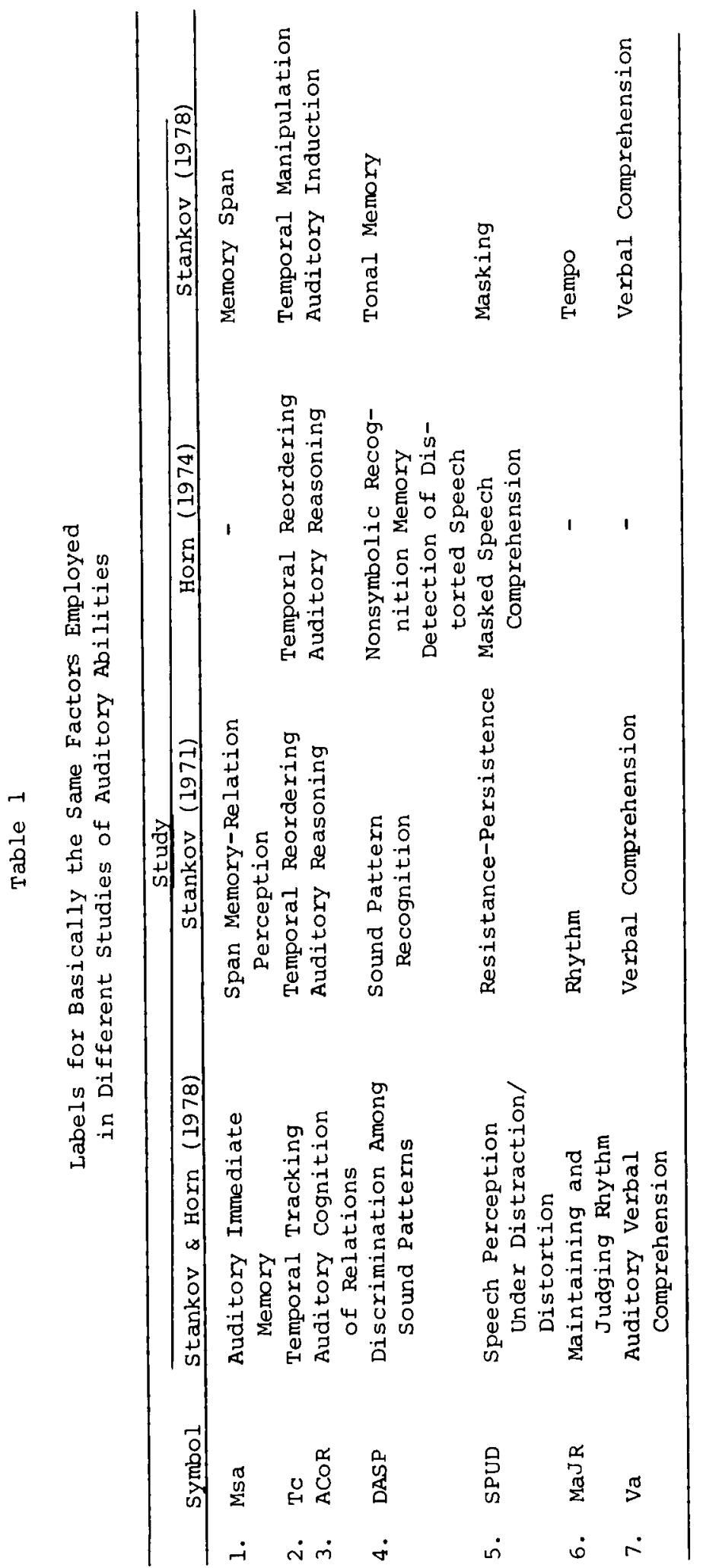




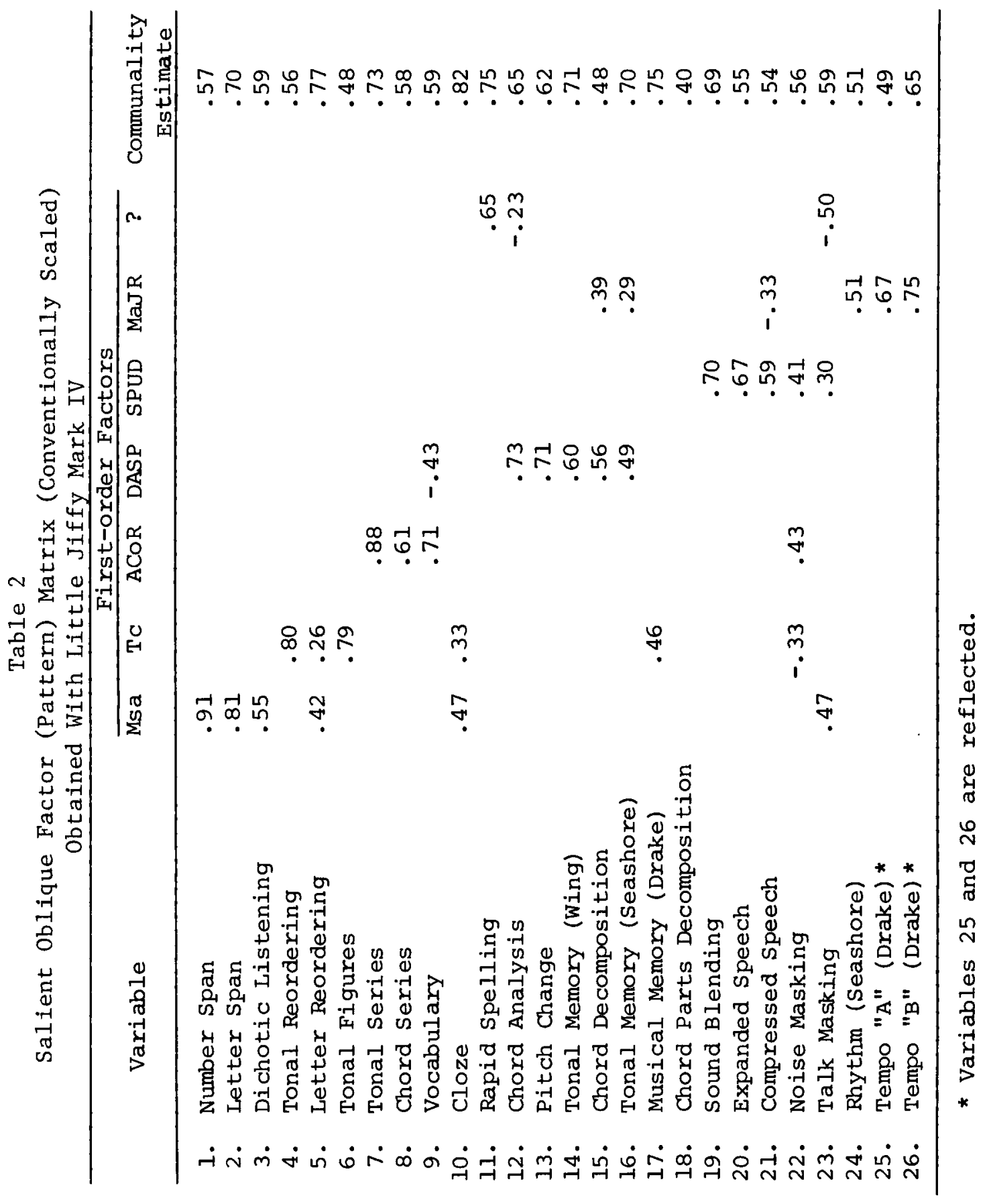


Stankov and Horn's Auditory Cognition of Relations (ACoR, Factor III) is identified in the present investigation by the high loadings on Tests 7 and 8-Tonal and Chord Series-which represent markers for inductive reasoning. However, in the present analysis Vocabulary loads .71 on this factor, implying that the present primary is broader than the original one. It is possible to argue that Vocabulary here might be measuring fluid intelligence. This test was given first, which means that it served as an example for introducing to all subjects new kinds of problems and a new and more difficult procedure for recording answers; it required clear understanding of the forthcoming task requirements. The loading of Vocabulary on the ACoR factor might come as a result of these effects, but the possibility cannot be ruled out that there was some systematic bias introduced in our procedure.

The only factor which was not replicated here was Auditory Verbal Comprehension. The seventh factor cannot be interpreted as Auditory Verbal Comprehension, since only one of three markers load on it (Test 11, Rapid Spelling). Rather, this factor should be considered as a specific with some variance shared with Talk Masking. In retrospect, it is possible Verbal Comprehension did not show up in the data mainly because of procedural errors. One possibility had already been mentioned in relation to the Vocabulary test, and the second is probably a function of the scoring of Rapid Spelling. (It will be recalled that blind subjects could use braille contractions and the sighted had to spell correctly.)

Second-Order analysis. Factor analysis of the primary factor intercorrelations produced only one general factor instead of three (Gf, Gc, and $\mathrm{Ga}$ ). This factor, as can be seen at the bottom of Table 3, had salient loadings on Auditory Immediate Memory, Auditory Cognition of Relations, and Temporal Tracking; but the other four primaries also had some (admittedly non-salient) loadings on it. The first three primaries are markers for fluid intelligence; and if the rationale of Little Jiffy is strictly followed, this appears to be a broad Gf factor. On the other hand, the theory regarding the secondorder factoring is poorly developed at present. For example, there is no rationale for the number of factors to be extracted, and it is quite possible that different criteria for salience could be advocated. Under the assumption that new criteria would have to be lowered, the second-order factor should be interpreted as "g." The implications of this finding will be considered in a later section of this paper.

In Stankov's (1978) study the first three primaries defined a $\mathrm{Gf}$ second-order factor. If the correlations of extension variables with these primaries are considered, similar interpretation can be achieved with the present data as well. It can readily be seen that the first three primaries

Table 3

Correlations Between Some Extension Variables and First Order Factors and Loadings on the Second Order Factor

\begin{tabular}{|c|c|c|c|c|c|c|c|}
\hline \multirow[b]{2}{*}{ Variables } & \multicolumn{5}{|c|}{ Primary Factors } & \multirow[b]{2}{*}{ MaJ R } & \multirow[b]{2}{*}{$?$} \\
\hline & Msa & TC & $\mathrm{ACOR}$ & DASP & SPUD & & \\
\hline Age & .31 & .21 & .14 & -.01 & .29 & .25 & .09 \\
\hline $\operatorname{sex}$ & -.23 & -.16 & -.02 & .07 & -.30 & -.08 & -.16 \\
\hline IQ (Verbal) & .38 & .48 & .46 & .28 & .29 & .25 & .33 \\
\hline Handedness & -.09 & -.06 & -.09 & .02 & -.05 & -.06 & -.10 \\
\hline $\begin{array}{l}\text { Second-order } \\
\text { factor }\end{array}$ & $.85 *$ & $.88^{*}$ & $.79 *$ & .64 & .75 & .70 & .66 \\
\hline
\end{tabular}

* Salients as indicated by Little Jiffy's criteria for salience. 
correlated $.38, .48$, and .46 with verbal estimates of IQ. Previous studies indicated that if the second-order factor were $\mathrm{Gc}$, the correlations of the primaries defining it with Verbal IQ should be higher than this. The above correlations are typically found when the relationship between $\mathrm{Gf}$ and $\mathrm{Gc}$ is studied.

The remaining four first-order factors loading on the general factor were DASP, SPUD, MaJR, and Spelling. Apart from Spelling, these primaries would be expected to load on the General Auditory Factor $(\mathrm{Ga})$. Their low correlations with Verbal IQ (in the .20s) implies that Gc is not measured by these primaries. It is most likely that if a second broad factor had come out of this data, it would have been $\mathrm{Ga}$ with loadings from Msa, SPUD, and MaJR. However, such a factor was not obtained, and an attempt to extract a factor of this kind would be contrary to Little Jiffy's rationale.

\section{Differences Between Sighted, Partially Sighted, and Blind.}

Equality of covariance matrices. The finding that factors of previous studies have largely replicated here with all 90 subjects does not necessarily mean that the factors are the same within the samples. There are two ways to check for this possibility: Factor analyses for each of the three samples can be carried out separately, or the homogeneity of covariance matrices among the samples can be tested. In the latter case, homogeneity would imply equality of factor structure (heterogeneity, however, would not necessarily imply differences). Since factor analysis of a 26 by 26 matrix based on 30 subjects can hardly command any respect, the second approach has been adopted. Bartlett's test for the homogeneity of covariances proved significant $[F(720 / 19611)=1.22]$, and the issue of factor structure similarity remains unresolved.

A definite answer to this question can only be obtained through the application of the first approach. Since the test of homogeneity of covariances includes diagonal elements which are variances, a different form of Bartlett's test can be used to check on homogeneity of variances. Eight out of 26 chi-square values were significant; therefore, at least some part of the heterogeneity of the covariances was caused by heterogeneity of the variances which do not affect factor structure. In the present context it is also important to note that there was no appreciable difference between the factor matrices obtained by analyzing the correlational matrix based on total scores and the pooled "within" groups correlational matrix.

Differences between mean vectors. Following is a summary account of the major results. (Means, standard deviations, and all other information regarding the analyses performed with the data can be obtained from the first author.) The question under consideration was, "Are the three mean vectors statistically different?" It has been stated before that this question can be answered through multivariate analysis of variance. The multivariate $F$-test for testing the differences between the mean vectors for the 26 variables $[F(62 / 114)=2.35 ; p<.05]$ indicated that overall performance of blind, partially sighted, and sighted subjects was significantly different. The nature of these differences needs to be considered further.

Inspection of the mean vectors for the three samples showed that the partially sighted subjects performed worse than blind and sighted children on all the tests. Sighted children were superior to blind (as found in post-hoc comparisons) on Vocabulary, Talk and Noise Masking, and Drake's Tempo B; and they were inferior on Rapid Spelling and Letter Span.

The partially sighted group's poor performance on all of the tests is probably due to the selection of these children for special schools. It is a policy of special schools for the partially sighted to encourage their pupils to return to normal schools as soon as possible. Typically, those remaining in special schools have either intellective and/or personality problems in addition to visual impairment. 
Table 4

Means for the Factor Scores for Sighted, Partially Sighted and Blind Subjects*

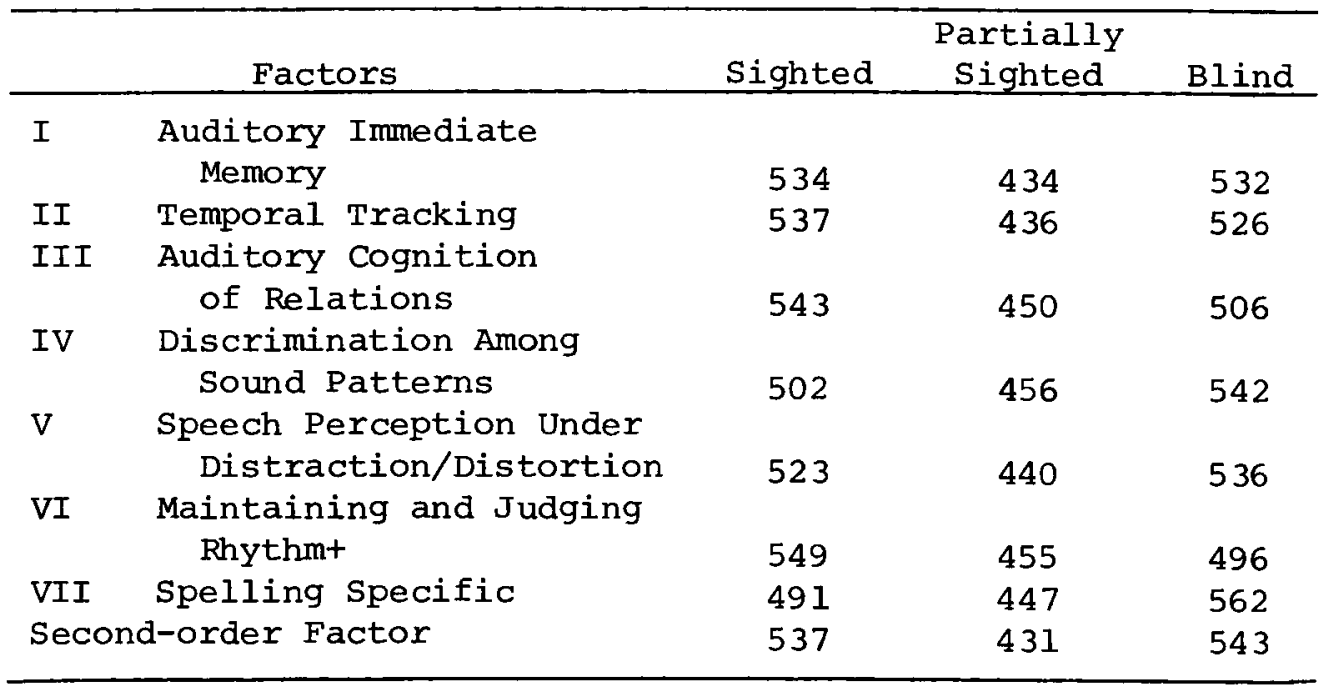

* Factor scores were scaled with mean of 500 and standard deviation of 100.

+ Scores for this factor are reflected.

It may be informative to relate the differences between the groups to scores on the factors obtained in the first part of this study. Table 4 gives means for the factor scores for the three groups on all factors. From that table it can be seen that the blind and sighted were almost the same on the second-order factor. (Although the blind children were superior on the seventh factor, Spelling specific, that is of little interest here.) Among the Gf primaries, blind and sighted children did not differ on Msa and Tc; but sighted appeared superior on ACoR. It should be recalled that $A C O R$ had loadings on Vocabulary and Noise Masking, both of which showed significant differences but were not proper markers for ACoR.

Vocabulary in the present context should be treated with some caution; the difference between sighted and blind children on this variable should be replicated again before considering it seriously. Although there may have been a genuine difference due to school curricula, it may be that there was a systematic bias in the administration of vocabulary tests, as mentioned earlier in this paper. (It should be noted that Verbal IQ scores did not indicate the same tendency; blind and sighted had the same average IQ score.) For that reason, it appears appropriate to say that there was virtually no difference between blind and sighted on fluid intelligence.

Regarding $\mathrm{Ga}$ primaries, there seem to be more grounds for claiming that there is difference. This particularly holds for the MaJR factor, on which sighted children excelled (due to their performance on Drake's Rhythm B), and DASP, on which blind children showed superiority. Concerning the latter, it can be said that the accumulation of small nonsignificant differences from all the variables involved, especially from Wing's Tonal Memory and Chord Decomposition, produced blind children's superiority. It should be noted that if a single score is obtained for $\mathrm{Ga}$, there may be no 
difference between the groups because of the opposing signs of the differences. Although sighted were better in both Masking tests, there did not appear to be any difference on SPUD-a consequence of a nonsignificant but opposite trend shown by other variables of this factor.

\section{Discussion}

The purpose of the present study was twofold. First, the intention was to replicate the findings of Stankov (1971) and Stankov and Horn (in press) regarding the factorial structure of auditory abilities. Considering changes in procedure and differences in the samples of subjects used, the replication achieved is remarkable. Of seven primary factors, six corresponded closely to the previous findings. These are Auditory Immediate Memory, Cognition of Auditory Relationships, Temporal Tracking, Discrimination Among Sound Patterns, Speech Perception Under Distraction/Distortion, and Maintaining and Judging Rhythm. The only factor which failed to replicate was Verbal Comprehension. At the second order, only one broad factor appeared.

Any comprehensive theory of human abilities should take account of the findings within the auditory modality. Temporal Tracking is a new primary of Gf; and SPUD, MaJR, and DASP form another broad factor representing perceptual cognitive processes of audition (Stankov, 1971). Studies of Karlin (1941,1942), Harris (1964), and Solomon (1959) identified factors similar to our DASP and SPUD ("Masking" being the preferred label for the latter). MaJR has been identified less often and Temporal Tracking is a completely new factor brought about by the applications of the present battery.

Stankov's $(1971,1978)$ and Horn and Stankov's (in prep.) results indicate that with the auditory tests, three second-order factors can be expected-Gf, Gc, and Ga. There are several possible reasons why they did not appear here. The first, and probably the least likely explanation, derives from the application of the Little Jiffy procedure of factoring. It is conceivable that the root-one criterion for the number of factors should not be applied at the higher orders. Likewise, it is known that Little Jiffy produces higher factor intercorrelations than the other rotated solutions (e.g., promax).

The second explanation follows from the selection of variables for this study and the obtained first-order structure. In particular, since Auditory Verbal Comprehension did not appear, crystallized intelligence was underdetermined by the other primaries, which might have some variance in it. While this might be a plausible explanation for merging $\mathrm{Gf}$ and $\mathrm{Gc}$, it does not explain why $\mathrm{Ga}$ did not emerge.

There is a third explanation for the present result. While it is possible that the presence of blind and partially sighted children in our sample could have caused different second-order structure, this is quite unlikely in view of Juurmaa's (1967) finding. On the other hand, the subjects in the present study were close in age to Stankov's (1978) subjects. In this latter study it was found that at the age of 11 to 12 , broad perceptual factors seem to be poorly defined; and, indeed, a completely differentiated ability structure typical of adults cannot be expected to appear.

All the primary abilities identified here can be applied in various situations in the same fashion as the other well-established abilities from the visual domain. The most natural application would be in investigating the performance of visually handicapped people, and the second aim of the present study was to explore the feasibility of this proposition. In particular, the aim was to investigate the differences in performance of the sighted, partially sighted, and blind.

On the basis of the evidence presented here, it is impossible to say whether factor structure would be the same in blind and partially sighted. A crude measure of homogeneity of covariances indicates that this is not the case. Juurmaa (1967) reports a more encouraging result; it is quite possible that further work will lead to the same conclusion with our tests as well. 
Present results indicate that partially sighted subjects-those with pronounced visual deficiency, but not to the extent of having to use braille in their school work-performed worse than either blind or sighted on all the tests of our battery. This has been attributed to their possible cognitive and/or personality problems in addition to those associated with poor vision. Teachers' experiences indicate that partially sighted are more "difficult to handle" than the blind.

Blind and sighted performed equally well on all three primaries of fluid intelligence, but interesting differences occurred with regard to $\mathrm{Ga}$ primaries. On the Discrimination Among Sound Patterns factor, the blind performed better than sighted (see also Pitman, 1965). On Maintaining and Judging Rhythm, they performed worse-a finding which agrees with Juurmaa (1967). Finally, they were not different from sighted on the Speech Perception Under Distortion/Distraction primary as measured here, but they were inferior on two Masking tests which consistently load on this primary. There appear to be no studies in which blind subjects were given Masking tasks; this finding appears to be contrary to intuition.

The finding with MaJR indicates that another popular belief does not have much firm ground: Instead of being better, blind subjects were actually worse than sighted subjects in perceiving and maintaining rhythm. Performance was especially poor when there was some kind of interference, such as in Drake's Tempo B. In other words, it appears that when presented with a multitude of sounds (such as at a cocktail party), the blind have pronounced trouble in perceiving relevant information or in maintaining steady inner tempo against which they can judge the temporal flow of events. At present it is not clear whether this should be attributed to (1) a perceptual difficulty; (2) personality traits of the blind; or (3) the interaction of these two.

It is clear that our tests can be used with visually handicapped. If further research shows that the factorial structure for this group is the same, the task of standardization can be readily undertaken.

\section{References}

Cooley, W. W., \& Lohnes, P. R. Multivariate data analysis. Sydney: Wiley, 1971.

Drake, R. M. Manual for Drake musical aptitude tests. Chicago: University of Chicago Press, 1954.

French, T. W., Ekstrom, R. B., \& Price, A. I. Manual for kit of reference tests for cognitive factors. Princeton NJ: Educational Testing Service, 1963.

Harris, T. D. A factor analytic study of three signal, detection abilities. Journal of Speech Hearing $R e$ search, 1964, 7, 71-78.

Hayes, S. P. Contributions to a psychology of blindness. New York: American Foundation for the Blind, 1941.

Horn, J. L. Organization of abilities and the development of intelligence. Psychological Review, 1968, 75, 242-259.

Horn, J. L. Theory of functions represented among auditory and visual test performances. In T. R. Royce (Ed.), Contributions of multivariate analysis to psychological theory. London: Academic Press, 1974.

Horn, J. L., \& Stankov, L. Human abilities revealed through visual and auditory tests. In prep.

Juurmaa, J. The ability structure of the blind and the deaf: Final report. American Foundation for the Blind Research Bulletin, 1967, 14, 109-122.

Kaiser, H., \& Rice, P. Little Jiffy, Mark IV. Educational and Psychological Measurement, 1974, 37, 111-117.

Karlin, J. E. Music ability. Psychometrika, 1941, 6. $61-65$.

Karlin, J. E. A factorial study of auditory function. Psychometrika, 1942, 7, 251-279.

Morse, T. The adaptation of a non-verbal abstract reasoning test for use with the blind. American Foundation for the Blind Research Bulletin, 1971, 23, 39-46.

Pitman, D. J. The musical ability of blind children. American Foundation for the Blind Research Bulletin, 1965, 11, 63-79.

Rice, C. E. Early blindness, early experience and perceptual enhancement. American Foundation for the Blind Research Bulletin, 1970, 22, 1-22.

Seashore, C. E., Lewis, D., \& Saetveit, J. C. Manual of instructions and interpretation for the Seashore measures of musical talents (2nd Ed.). New York: Psychological Association, 1960. 
Shuter, R. The psychology of musical ability. London: Methuen, 1968.

Solomon, L. Search for physical correlates to psychological dimensions of sounds. Journal of Acoustical Society of America, 1959, 31, 492-497.

Stankov, L. The hierarchical structure of auditory abilities and the relationship between auditory and visual modalities. Unpublished doctoral dissertation, University of Denver, 1971.

Stankov, L. Fluid and crystallized intelligence and broad perceptual factors among the 11-12 year olds. Journal of Educational Psychology, 1978, 70. 324-334.

Stankov, L., \& Horn, J. L. Human abilities revealed through auditory tests. Journal of Educational Psychology, in press.
Wing, H. Manual for standardized tests of musical intelligence. Sheffield, England: City of Sheffield Training College, 1966.

\section{Acknowledgments}

We are grateful to Associate Professor J. A. Radcliffe and participants of the Individual Differences Seminar at the University of Sydney for many valuable comments regarding the earlier draft of this paper. We also benefited from the discussions with Professor J. L. Horn at the University of Denver.

\section{Author's Address}

Lazar Stankov, Department of Psychology, University of Sydney, Sydney N.S.W. 2006, Australia. 